\title{
RECENT DEVELOPMENTS IN THE DIAMOND STORAGE RING DESIGN
}

\author{
J. A. Clarke, D. J. Holder, J. K. Jones, H. L. Owen, M. W. Poole, D. J. Scott, S. L. Smith, \\ J. A. Varley \& N. G. Wyles, CLRC Daresbury Laboratory, Warrington, UK
}

\section{Abstract}

The Design Phase of DIAMOND, a $3^{\text {rd }}$ generation light source facility, is currently in progress. Earlier studies of a similar storage ring had found a basic lattice structure with low emittance, good dynamic stability and the flexibility to operate in different modes. The lattice has been further developed to meet the evolving demands of the project, delivering very high brightness radiation over a wide photon energy range. Optimisation of the lattice properties, including those of the non-linear dynamics, is described in detail, together with other general characteristics of the storage ring.

\section{OPTICAL STRUCTURE}

Changes to the user requirements for straight length and approval of the key parameters for the storage ring have led to significant recent development in the lattice layout; details of the overall project are given in a separate paper [1]. In common with other $3^{\text {rd }}$-generation light source designs, the challenge is to satisfy the conflicting requirements of reaching low emittance (for high brightness output), whilst maintaining flexibility in the provision of ID straights, adequate dynamic aperture for injection and beam lifetime, an and economic overall circumference.

\subsection{Storage Ring Layout and Design}

To satisfy user requirements, the present design comprises a double-bend achromatic structure which is detuned to give finite dispersion in the insertion device (ID) straight sections, resulting in a smaller natural emittance around $2 \mathrm{~nm}$-rad. A 6-fold periodic structure has been chosen, with one each of two $8 \mathrm{~m}$ long straights being used respectively for injection and RF, and 4 other $8 \mathrm{~m}$ straights for long undulators (the $8 \mathrm{~m}$ free space requirement is principally set by the requirement to fit in 3 CESR-type superconducting cavities [2]). To provide the optimum betatron functions for other insertion devices and to restrict the overall circumference $5 \mathrm{~m}$ free space is available in the other ID straights.

Two families of sextupoles at points of high dispersion within the pseudo-achromat perform most of the chromatic correction, with 4 additional families performing the remaining correction and correcting for non-linear effects [3]. Additional windings on these magnets will provide both slow orbit correction and skew quadrupole fields to control coupling effects, minimising the required circumference. Fast correction up to probably $100 \mathrm{~Hz}$ will be provided by 4 dedicated correctors adjacent to each ID straight. Significant effort has been made to minimise the amount of space required, resulting in an overall design circumference of $560.4 \mathrm{~m}$.

The small target emittance is only readily achieved by the use of non-zero dispersion in the straights [3], the emittance and small dispersion within the pseudoachromat necessitating strong sextupoles and their consequent limiting effects on the dynamic aperture. Phase advances between each family of sextupoles has been set to $5 \pi / 2$ horizontally and $\pi$ vertically to provide cancellation of non-linear kicks [3], the long straights allowing some flexibility in setting the overall tune. The tune is presently set a little above the integer in both planes to avoid resistive-wall problems.

The linear optical functions are shown in Figure 1, with working point shown in Figure 2. The principal lattice parameters are given in Table 1.

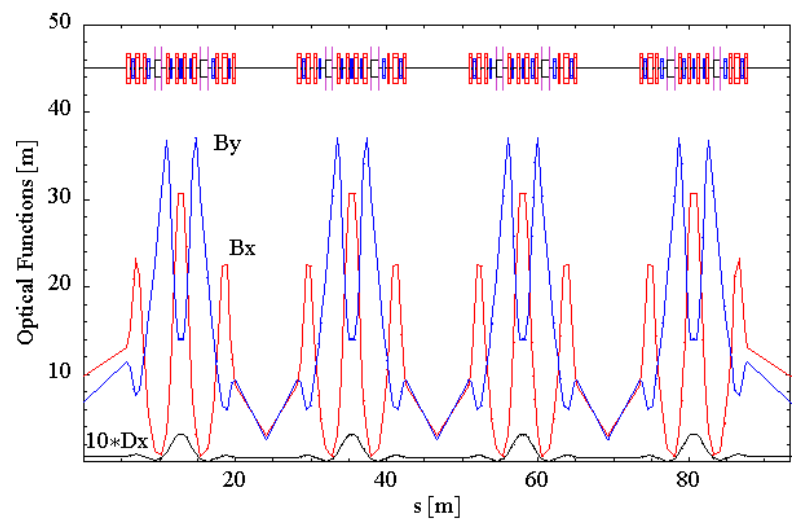

Figure 1: Betatron functions and horizontal dispersion over one superperiod.

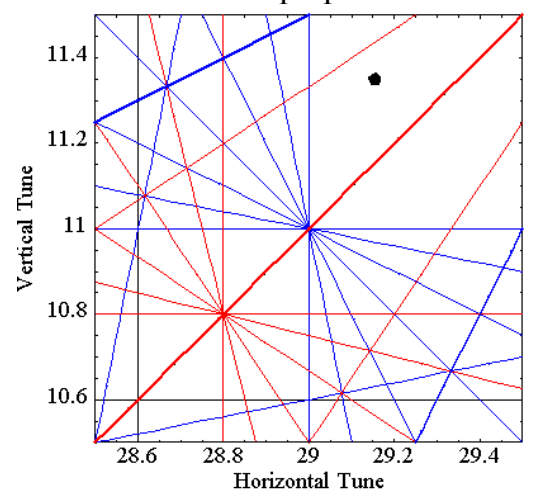

Figure 2: Working diagram in the neighbourhood of the chosen tunes, showing only structural resonances. 
Table 1: Principal DIAMOND Parameters

\begin{tabular}{|l|c|}
\hline Energy & $3 \mathrm{GeV}$ \\
\hline Beam current & $300 \mathrm{~mA}$ \\
\hline No. of DBA cells & 24 \\
\hline Symmetry & 6 \\
\hline Circumference & $560.4 \mathrm{~m}$ \\
\hline Harmonic no. (500 MHz) & 934 \\
\hline ID space available & $18 \times 5 \mathrm{~m}, 4 \times 8 \mathrm{~m}$ \\
\hline Dipole field & $1.4 \mathrm{~T}$ \\
\hline Emittance & $2.0 \mathrm{~nm}-\mathrm{rad}$ \\
\hline Coupling & $1 \%$ \\
\hline Betatron tunes & $29.16,11.35$ \\
\hline Natural chromaticity & $-100,-42$ \\
\hline Dispersion: long,short sts & $6.5,5.1 \mathrm{~cm}$ \\
\hline Momentum compaction & $1.45 \times 10^{-4}$ \\
\hline Natural energy spread & $9.61 \times 10^{-4}$ \\
\hline
\end{tabular}

\subsection{Non-Linear Optimisation}

Since the sextupole families are interleaved complete cancellation of their non-linear kicks is not possible, and harmonic sextupoles must be used to provide adequate dynamic aperture. Optimisation of the harmonic strengths is based upon the minimisation of tune shifts with amplitude and momentum, since it is most likely that the crossing of resonances at large amplitudes is the primary effect limiting the dynamic aperture [4]; the resulting tune footprint in the working diagram then guides the choice of working point. In the present design 4 families of harmonic sextupoles have been used to optimise the dynamic aperture and momentum acceptance. The target apertures are such as to provide at least 10 hours lifetime in multibunch mode (with $2 / 3$ of r.f. buckets uniformly filled); this leads to a target dynamic aperture of $\pm 20 \mathrm{~mm}$ horizontally and $\pm 10 \mathrm{~mm}$ vertically in the long straights ( $\beta$-function of $9.8 \mathrm{~m}$ and $6.8 \mathrm{~m}$ respectively), and a momentum acceptance of $\pm 4 \%$ (see below).

Using the working point above, these dynamic apertures have been achieved to date, but further work is required to optimise these (see Figure 3).

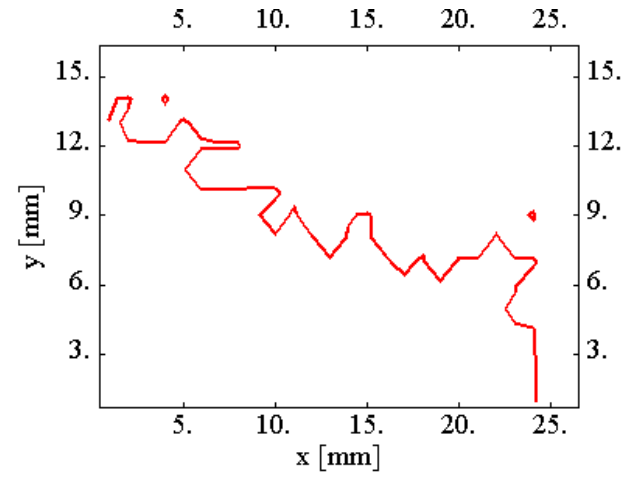

Figure 3: On-momentum dynamic aperture.

\section{SINGLE PARTICLE EFFECTS}

\subsection{Vertical Momentum Acceptance}

The small vertical apertures required in the ID straight sections for high brightness - as little as $7 \mathrm{~mm}$ internally in the case of some $2 \mathrm{~m}$ devices - can present a restriction on the momentum acceptance due to the motion from Touschek scattered particles being coupling into the vertical plane. Non-linear effects at these large amplitudes can give this halo of scattered particles a coupling much larger than that of the core of the beam. The actual halo coupling value must in general be estimated numerically via particle tracking [5], and is sensitive to the exact arrangement of sextupoles. However, estimates have been made for the present lattice (Figure 4) which show that even with full coupling in the scattered halo the resulting momentum acceptance from the vertical apertures are larger than those from dynamical, RF and horizontal aperture restrictions.

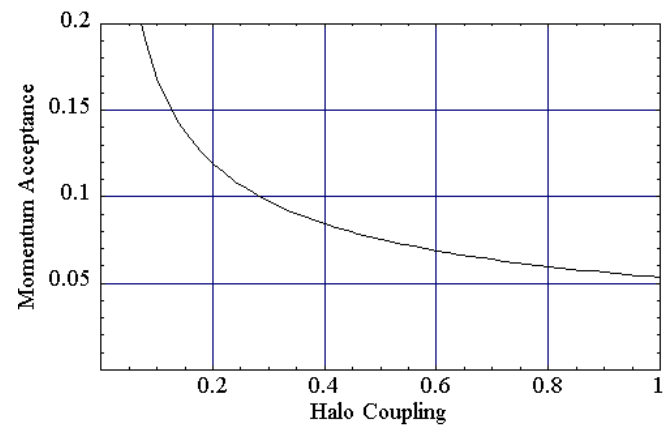

Figure 4: Momentum acceptance as a function of halo coupling.

\subsection{Emittance Ratio and Coupling Correction}

The specified brilliance values assume the achievement of $1 \%$ coupling of the natural emittance in the vertical plane. To ensure this the alignment tolerances of elements are specified as shown in Table 2, and skew quadrupole fields will be available from the sextupole magnets to allow control of the coupling value. The calculation method is described in Ref. [6]. The emittance ratio from vertical dispersion and betatron coupling is shown in Table 3.

Table 2: Dominant alignment errors.

\begin{tabular}{|l|c|}
\hline Dipole Roll Error & $0.2 \mathrm{mrad}$ \\
\hline Quadrupole Roll Error & $0.2 \mathrm{mrad}$ \\
\hline Quadrupole Position & $0.1 \mathrm{~mm}$ \\
\hline Sextupole Position & $0.1 \mathrm{~mm}$ \\
\hline
\end{tabular}

Table 3: Summary of emittance ratio effects .

\begin{tabular}{|l|c|}
\hline Ave. Vertical Dispersion & $1.4 \mathrm{~cm}$ \\
\hline$\varepsilon$ Ratio from Vert.Disp. & $0.2 \%$ \\
\hline Betatron Coupling & $0.65 \%$ \\
\hline Total $\varepsilon$ Ratio & $0.87 \%$ \\
\hline
\end{tabular}




\section{MULTI-PARTICLE EFFECTS}

\subsection{Beam Lifetime}

Both Touschek and gas scattering effects will be significant in both the multibunch and single bunch operating modes, gas scattering becoming dominant in $2 \mathrm{~m}$ short-period IDs at vacuum apertures less than $\pm 3.5 \mathrm{~mm}$. The sensitivity of the Touschek lifetime to impedance effects via the bunch length makes the lifetime complex to predict, and thus we have been conservative in specifying a momentum acceptance of $4 \%$. The broadband impedance from r.f. cavities will arise primarily from the matching tapers, and is not yet known, therefore the lifetimes given in Table 4 are estimates based on an overall impedance of $1.3 \Omega$ and a representative possible complement of IDs, using ZAP [7]. $3^{\text {rd }}$-harmonic cavities are being considered as a possible method for increasing the lifetime via bunch lengthening.

Table 4: Lifetime estimates, assuming 300mA, 2/3 bunches filled in multibunch mode, and $10 \mathrm{~mA}$ bunch current in single bunch mode. SPEAR scaling is assumed.

\begin{tabular}{|l|l|}
\hline Multibunch Touschek & 36 hours \\
\hline Single Bunch Touschek & 16 hours \\
\hline Gas Scattering & 34 hours \\
\hline Multibunch Total & 18 hours \\
\hline Single Bunch Total & 11 hours \\
\hline
\end{tabular}

\subsection{Instability Thresholds}

Using the estimated impedance as above a ZAP calculation has been made of the threshold current for the onset of the microwave instability and this gives a threshold (without SPEAR scaling) of $13 \mathrm{~mA} / \mathrm{bunch}$. At the moment we are assuming an operating single bunch user requirement of around $10 \mathrm{~mA}$. Longitudinal and transverse HOM thresholds are well beyond the highest DIAMOND operating current of $300 \mathrm{~mA}$; the calculations suggest that the choice of superconducting CESR cavities means that longitudinal instability feedback will not be needed and transverse feedback will only be necessary to counter resistive wall effects.

\subsection{Fill Structure and Ion Effects}

Hydrogen, Carbon Monoxide and Carbon Dioxide are the dominant residual gas species likely to be present in the storage ring; Helium, Methane, Water, Neon, Nitrogen, Oxygen and Argon may also be present in significant amounts, the isotopes of which may be trapped by the focusing effect of the passing bunch trains. The possibility of ion trapping has been estimated for these species [8], showing that trapping can occur over a wide range of gap sizes and beam currents (Figure 5). However, above a gap size of 214 bunches trapping occurs for $5 \%$ of operating beam currents or less, and this is taken as a minimum operating gap size.

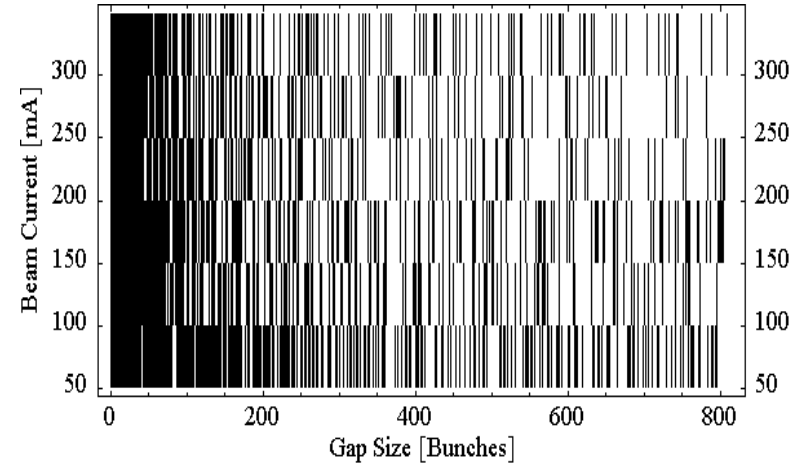

Figure 5: Trapping of any significant residual ion species as a function of gap size in the bunch train and overall beam current (a black bar indicates trapping).

\section{FUTURE PLANS}

Further work is required to refine the sextupole scheme for on- and off-momentum particles with closed-orbit errors, and to develop the fast orbit control, injection, feedback and coupling control arrangements. Outlines for all of these systems will be made as part of the present ongoing design study.

\section{REFERENCES}

[1] J.A.Clarke et. al. 'The DIAMOND Project - An Advanced Light Source for the UK', this conference.

[2] D.Moffat et al., 'Preparation and Testing of a Superconducting Cavity for CESR-B', PAC'93, Washington, May 1993.

[3] A.Wolski, 'Optimisation of the Dynamic Aperture of the DIAMOND Storage Ring Lattice', EPAC 2000, Vienna, June 2000

[4] P.Nghiem et al., 'Optics for SOLEIL at $2.5 \mathrm{GeV}$ ', PAC'97, Vancouver, May 1997.

[5] M.Böge and A.Streun, 'Beam Lifetime Studies for the SLS Storage Ring', PAC’99, New York, March 1999.

[6] M.Muñoz, 'Assessment of the Achievable Emittance Ration in DIAMOND', EPAC'96, Sitges, June 1996.

[7] M.S.Zisman et al., 'ZAP User's Manual', LBL-21270 (1986).

[8] C.J.Bocchetta and A.Wrulich, Nucl.Inst.Meth. A278, 807 (1989) 